\title{
Investigation of the impact of hot-carrier-induced interface state generation on carrier mobility in nMOSFET
}

\author{
Zhicheng Wu, Graduate Student Member, IEEE, Jacopo Franco, Member, IEEE, Brecht Truijen, Philippe \\ Roussel, Ben Kaczer, Dimitri Linten, and Guido Groeseneken, Fellow, IEEE
}

\begin{abstract}
A comprehensive investigation on the hot carrier induced interface state generation and its impact on carrier mobility in nMOSFET is performed. I-V compact modelling and charge pumping characterization are used as independent ways to evaluate the interface state density as a function of hot carrier induced aging. From the two techniques, similar power-law time exponents of the interface state density kinetics are obtained. Assisted by the quasi-spectroscopic (temperature-resolved) charge pumping measurement, the extracted interface state density is further correlated with the I-V modelling results: an universal mobility degradation normalization parameter $N_{i t \text {,ref }}=\sim 4.1 \times 10^{11} / \mathrm{cm}^{2}$ is reported, irrespective of the effective-oxide-thickness (EOT), stress temperature, or the relative degradation of the device-under-test (DUT). Supported by the fundamental principles deployed in the derivation as well as the broad range of experimental conditions considered for its validation, the reported normalization parameter could serve as a modelling constant in the commonly used I-V compact models to correlate the mobility degradation with the interface state density induced by hot carrier stress.
\end{abstract}

Index Terms-Charge pumping, hot carrier degradation, MOSFET, compact model, reliability, electron mobility, interface state

\section{Introduction}

$\mathrm{A}^{\mathrm{s}}$ $\mathrm{S}$ transistor's dimensions shrink without a corresponding reduction of the supply voltage, hot carrier degradation is becoming one of the main reliability concerns for state-of-theart nMOSFETs [1,2]. Both TCAD- [3,4] and compact-modelbased strategies $[5,6,7]$ have been demonstrated for modelling the hot carrier induced device degradation. However, among all these efforts, no detailed investigation of the crucial correlation between the generated interface states and mobility degradation has been reported. Historically, different numerical forms have been used to translate a given interface state density into the corresponding mobility degradation $[8,9,10,11,12]$. Different proportionality relations have been used in these works, and no systematic match between experiments and models has been consistently demonstrated. While an accurate correlation between the interface state density and mobility degradation is clearly crucial, it is particularly challenging to establish given the combined effect of interface states on the device threshold

This paragraph of the first footnote will contain the date on which you submitted your paper for review.

Zhicheng Wu and Guido Groeseneken are with IMEC, 3001 Leuven, Belgium, and also with ESAT-MICAS, KU Leuven, 3001 Leuven, Belgium (e-mail: zhicheng.wu@imec.be). voltage. Furthermore, establishing such correlation is crucial also for unambiguously decoupling the threshold voltage shifts induced by generated interface states and trapped oxide charge $[13,14,15]$. A lack of experimental validation of the consistency between the interface state density extracted by modelling the $\mathrm{I}-\mathrm{V}$ curves and the physical defect density actually present in the aged device as probed, e.g., by charge pumping technique, may weaken any hot carrier degradation modelling effort. Therefore, establishing the correlation between hot carrier induced interface states and the corresponding mobility degradation is of fundamental importance for understanding and modelling hot carrier aging.

In this work, a systematic evaluation of hot carrier induced MOSFET degradation is performed, with focus on exploring the relation between carrier mobility degradation and interface state density: for the former, the charge-based EKV model $[16,17]$ is used to fit the aged device I-V characteristics; for the latter, temperature-resolved charge pumping measurements are performed to estimate the total interface state density $[18,19,20]$. A consistent power-law time exponent is observed for both the interface state density as estimated by charge pumping and for the mobility degradation ratio as estimated by calibrating the EKV model; based on this agreement, a normalization relation between interface state density and mobility degradation ratio is established, and a single normalization parameter is reported for all the investigated experimental conditions and device technologies. These results demonstrate unambiguously that a single modelling equation with a universal normalization parameter can be used to translate the hot carrier induced interface state into the mobility degradation. This approach can be deployed in device aging compact models for circuit simulations.

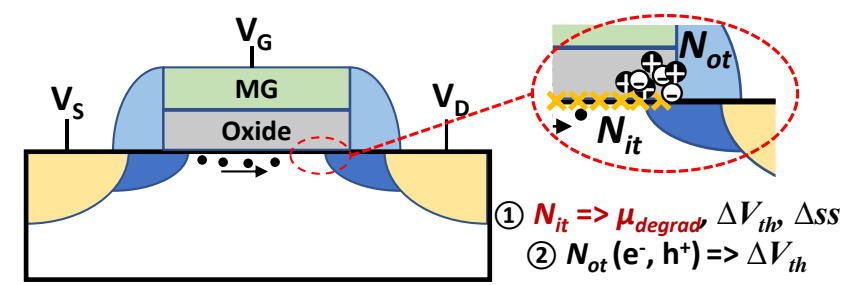

Fig. 1: Schematic representation of the hot carrier induced damage in the MOSFET, which typically localizes near the drain side due to the carrier energy peak there. Inset shows the hot carrier induced interface state $N_{i t}$ and oxide charge trapping $N_{o t}$ : for the latter one, both electron

Jacopo Franco, Brecht Truijen, Philippe Roussel, Ben Kaczer, and Dimitri Linten are with IMEC, 3001 Leuven, Belgium. 
and hole (from impact ionization) trapping can occur, depending on the carrier energy and local oxide field.

\section{I-V MODELLING}

Hot carrier degradation, driven by the high energy carrier near the drain side of the channel [21], causes non-uniform degradation in the MOSFET. A schematic representation of the hot carrier induced localized damage in a MOSFET is shown in Fig. 1, where both hot carrier induced interface state $\left(N_{i t}\right)$ and oxide charge trapping $\left(N_{o t}\right)$ are highlighted. The impact of hot carrier induced $N_{i t}$ is two-fold: first, it degrades the carrier mobility by introducing additional Coulomb scattering center; second, it affects the device electrostatics by shifting the threshold voltage and degrading the subthreshold slope. The localized nature of hot carrier degradation, combined with the kinetics of the defect density during stress, further complicates the interpretation of its impact on the device I-V characteristic. As the first step for correlating a given defect profile with the corresponding aged device $\mathrm{I}-\mathrm{V}$ characteristic, in the next Section the connection between defect density and corresponding I-V modelling parameters is established.

\section{A. The I-V model and its implementation}

The charge-based EKV model [16,17] employed here to describe the device I-V is briefly introduced. The normalized pinch-off potential $v_{p}$ and the normalized source and drain potential $v_{S, D}$ are defined in Eqs. (1-2):

$$
\begin{aligned}
& v_{p}=\left(V_{G}-V T H\right) /(N I D E \cdot u t) \\
& v_{S, D}=V_{S, D} / u t \text { with } u t=k T / q
\end{aligned}
$$

Where $k$ is the Boltzmann constant, $T$ is the temperature in Kelvin and $q$ is the elementary charge. NIDE is the so-called ideality factor and mainly influences the device subthreshold current. NIDE can be converted from the subthreshold slope as $N I D E=s s /[u t \cdot \ln (10)]$. As the solution of the "charge-potential linearization" [16], the gate, drain, and source voltage are connected to the normalized charge $q_{S, D}$ through the Lambert$\mathrm{W}$ (also known as product logarithm) function in Eq. (3).

$$
\begin{gathered}
q_{S, D}=0.5 \cdot \operatorname{lambertw}\left[2 \exp \left(v_{p}-v_{S, D}\right)\right] \\
i_{f, r}=q_{S, D}^{2}+q_{S, D}
\end{gathered}
$$

By applying Eqs. (3-4) separately to the gate-to-source (forward current $i_{f}$ ) and gate-to-drain (reverse current $i_{r}$ ) sides, the drain current (5) is calculated as the difference between forward $i_{f}$ and reverse current $i_{r}$, multiplied by the current normalization factor $\left(2 \cdot N I D E \cdot u t^{2} \cdot C_{o x} \cdot W / L\right)$ and carrier mobility $(\mu)$. Such normalization relation helps in decoupling transistor sizes or technology dependence, as the normalized drain current is only a function of the device bias voltages $\left(V_{G}, V_{D}, V_{S}\right)$, electrostatic control (NIDE) and temperature.

$$
I_{D}=2 \frac{W}{L} N I D E \cdot u t^{2} C_{o x} \mu\left(i_{f}-i_{r}\right)
$$

Under strong inversion condition, where $v_{p}-v_{S, D} \gg 1$, Eqs. (45) can be further simplified into Eqs. (6-7) [16]:

$$
\begin{gathered}
i_{f, r}=\left[\left(v_{p}-v_{S, D}\right) / 2\right]^{2} \\
I_{D}=\frac{W}{L} N I D E \cdot u t^{2} C_{o x} \mu\left(v_{D}-v_{S}\right)\left[v_{p}-\left(\frac{v_{D}+v_{S}}{2}\right)\right]
\end{gathered}
$$

Based on Eq. (7) and specifically to the hot carrier generated interface state $N_{i t}$, several conclusions can be drawn: first, the impact of $N_{i t}$ on carrier mobility $\mu$ enters as a multiplication factor and will degrade the device transconductance; second, the impact of $N_{i t}$ on the threshold voltage $V T H$ enters the pinch- off potential $v_{p}$ and will only shift the I-V and transconductance parallelly; third, since the pinch-off potential $v_{p}$ has already been normalized by NIDE [this is to reflect the gate electrostatic control over the channel, see Eq. (1)], the impact of $N_{i t}$ on NIDE only results in a slightly rescaled $\left(v_{D}+v_{S}\right) / 2$ (right term inside the square bracket of Eq. 7). For a linear transfer characteristic $\left(V_{D}=0.05 \mathrm{~V}\right)$ and under strong inversion $\left.\left(v_{p^{-}}-v_{S, D} \gg\right) 1\right)$, such impact is small. From above discussion and for the aged state, only the linear transfer characteristic under strong inversion will be modelled and only the $N_{i t}$ impact on $\mu$ and $V T H$ needs to be considered.

Furthermore, to account for the impact of $N_{i t}$ on Coulomb scattering limited mobility (cf. ECO term), a bias dependent mobility model is introduced, whereas for all other scattering mechanisms, e.g., phonon-limited and surface-roughness limited scattering, a single power-law term is adopted to capture the lumped effect (cf. EMOB term). The mobility representation is listed in Eqs. (8-9).

$$
\begin{gathered}
E_{\text {eff }}=-\left(Q_{d}+\theta \cdot Q_{i}\right) / \varepsilon_{s i} \\
Q_{i}=N I D E \cdot u t \cdot C_{o x} \cdot q_{s}
\end{gathered}
$$

Where $E_{\text {eff }}$ is the effective field in silicon, and $\theta$ is the weighting factor for the inversion charge and equals 0.5 for electron [22]. Using Matthiessen's rule, the final mobility expression is obtained in Eq. (10).

$$
\mu=1 /\left[\left(\frac{E_{e f f}}{E C O}\right)^{-1}+\left(\frac{E_{e f f}}{E M O B}\right)^{p m o}\right]
$$

Where $E C O=E C O_{0}$ for pristine device. As described above, the hot carrier induced interface states will introduce additional Coulomb scattering centers and therefore modifying ECO. Such physical effect is addressed by the mobility degradation ratio $K$ as defined below in Eqs. (11-12), which is then fed back to Eq. (10) to capture the degraded mobility $\mu$.

$$
\begin{gathered}
E C O=E C O_{0} \cdot(K+1) \\
K=N_{i t} / N_{i t, r e f}
\end{gathered}
$$

With increasing interface state density $N_{i t}$ (e.g., generated by hot carrier stress), the Coulomb scattering term in Eq. 10 (left term in the denominator) becomes evens larger, and therefore the mobility $\mu$ within the Coulomb scattering-limited regime becomes smaller (note the negative exponent for the Coulomb scattering term). Such relation originates from the proportional relation between Coulomb scattering limited mobility and mean momentum relaxation time, which is inversely proportional to the defect density [23]. The modelling equations have also been widely used in $[9,10,11,12]$. It is worth to emphasizing again that the target of this work is to validate this mobility degradation relation experimentally, as well as to explore and to establish the mobility degradation normalization parameter $N_{i t, \text { ref. }}$

To summarize this Section, the EKV model and its simplification under strong inversion are introduced, together with the physical mobility expression. Without considering the localization effect, the main feature of hot carrier degradation can be readily incorporated into the I-V model. For capturing the fresh linear transfer characteristic, 4 parameters (VTH, $E C O_{0}, E M O B, p m o$ ) need to be calibrated against measured I$\mathrm{V}$ data of the pristine device. By combining Eqs. 1, 2 and 7, it can be seen that any NIDE variation only results in a slightly rescaled $\left(v_{D}+v_{S}\right) / 2$ (i.e., the right term inside the square bracket of Eq. 7) and has no impact on the gate electrostatics (through 
$v_{p}$ ) under strong inversion; NIDE is therefore fixed to its pristine value calculated from the subthreshold swing ( $s s$ ) without further adjustment. For reproducing the aged linear transfer characteristic under strong inversion, only 2 parameters $(E C O$, $V T H$ ) are modified: notice that only $N_{i t}$ will degrade Coulomb scattering and thus the transconductance, and therefore the proposed calibration scheme is inherently robust against parameter covariance.

\section{$B$. The localized degradation and its equivalence}

The other crucial aspect of hot carrier damage is its strong localization as depicted in Fig. 1, which significantly complicates its physical understanding and modelling. To address this issue and to enable correlating the I-V modelling with experimental techniques that directly probe the interface defects, e.g., charge pumping, in the following, a simple transistors chain model is used to demonstrate the equivalence between the degradation caused by a localized $N_{i t}$ profile and a uniformly distributed $N_{i t}$ profile for what concerns the mobility degradation ratio $K$.

The model of a single transistor in linear regime under strong inversion adopts Eq. (7); to formulate the drain current of the transistor chain, a current sensitivity model is considered following Eq. (13), where $N$ is the number of identical transistors in the chain:

$$
I_{D}=1 /\left[N \cdot\left(\frac{1}{I_{D, 1}}+\frac{1}{I_{D, 2}}+\cdots+\frac{1}{I_{D, N}}\right)\right]
$$

Based on the discussion above, two representative cases are considered. In the first case, only $V_{t h}$-shift with different degree of localization is explored, whereas a fixed total degradation is maintained. As can be seen in Fig. 2, the nominal degradation of the "entire" device (transistors chain) depends on the degree of localization: the stronger the localization effect, the more the drain current degrades. Such result clearly indicates that the nominal $V_{t h}$-shift fitted from the I-V model, only reflects an apparent $V_{t}$-shift of the aged device, which is closely coupled to the localization effect.

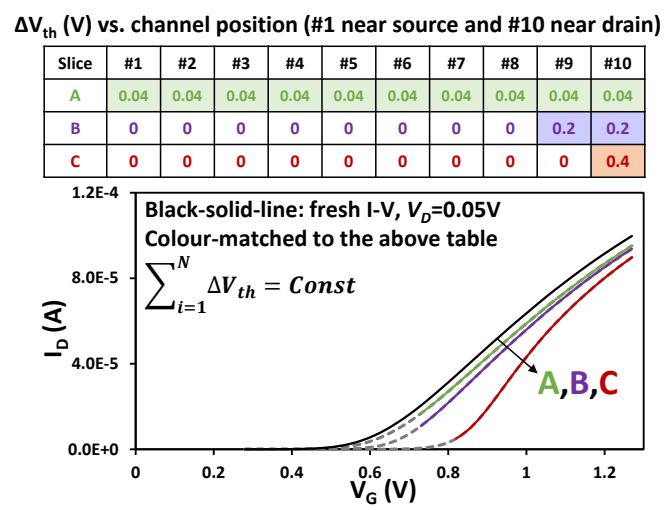

Fig. 2: $V_{t h}$-shift calculations by using the transistor chain model (10 identical transistors): a uniform $V_{t h}$-shift (case $A$ in green) results in the smallest current degradation, whereas the strong localized degradation (case C in red) results in the largest degradation. The "deformation" in curve $C$ is due to the large degradation of a single slice. Note: the solidline represents the modelling results in strong inversion, whereas the dashed-line represents the entire I-V curve (only for reference).

The second case is the mobility degradation ratio $[K$, as defined in Eqs. (11-12)], where the mobility is degraded, and a constant total degradation is maintained. The result, as summarized in Fig. 3, clearly indicates the overall mobility degradation is completely insensitive to the localization effect, and only reflects the "integrated" degradation. This key property will be exploited for exploring the mobility degradation under hot carrier stress, and to correlate it with charge pumping measurement results.

The difference between $V_{t h}$-shift and mobility degradation under different localization conditions can be understood as a direct result of the transistor chain and current sensitivity models (Eqs. 7 and 13). In fact, such positional sensitivity to interface states, which degrade carrier mobility, can be verified experimentally by measuring the forward and reverse (swapped Source/Drain) current degradation of the linear transfer curve under strong inversion, as exemplified in Fig. 4: for a given interface defect density generated under hot carrier stress, the linear current degradation is the same both for the case where defect-induced carrier scattering takes place at the source side (i.e., when carriers enter the channel region, cf. reverse current degradation), or for the case where such scattering takes place at the drain side (i.e., when carriers have travelled already along the channel, cf. forward current degradation) beyond which carriers are collected by the drain terminal. In fact, virtually no difference is observable between the forward and reverse linear current degradation. This observation readily confirms that the linear transfer curve under strong inversion is intrinsically insensitive to the relative defect location along the channel, whereas the defect density is the dominant factor for mobility (and thus linear current) degradation.

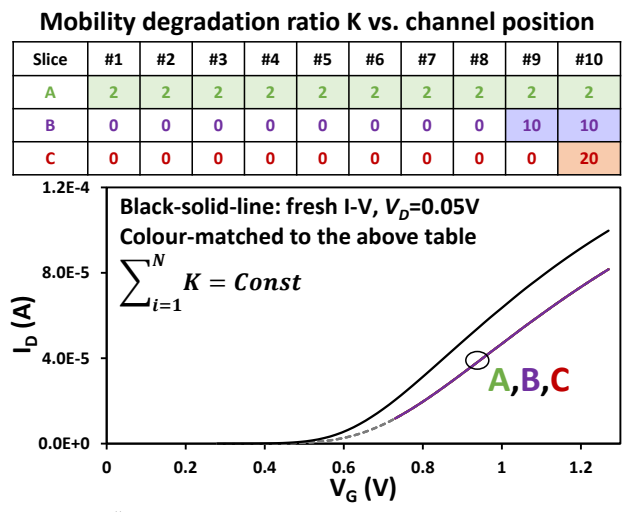

Fig. 3: The same "slicing" approach is applied to explore the mobility degradation. In contrast to the $V_{t h}$-shift (Fig. 2), the mobility degradation ratio shows no dependence on the damage localization. Therefore, the average degradation extracted from the I-V model can be directly used.

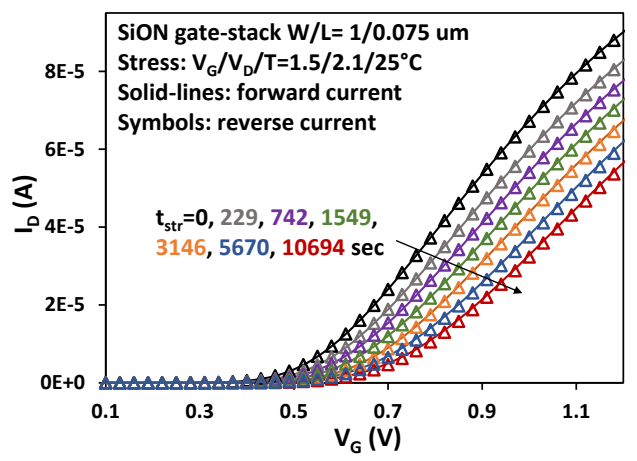

Fig. 4: Experimentally measured forward and reverse current degradation on device with SiON gate-stack (cf. device-B below), i.e., by swapping the bias $(0.05 \mathrm{~V}, 0 \mathrm{~V})$ between source and drain terminals. The negligible difference between forward and reverse current 
degradation effectively supports the simulation results in Fig. 3, where the linear transfer characteristic under strong inversion is intrinsically insensitive to the defect localization.

\section{Experiment}

Following the model-based study presented in the previous Section, a wafer-level stress-measure experiment is performed with the goal of generating different degree of interface damage, including different degree of localization as well as different interface state density, which is then quantified by the charge pumping technique and is further correlated with I-V modelling results. For this purpose, two types of planar nMOSFET with gate-stack optimized to minimize oxide trapping are used. The $V_{t h}$-shift under bias-temperature stress for device-A $\left(V_{G}=1.8 \mathrm{~V}, V_{D}=0.05 \mathrm{~V}, \mathrm{~T}=125^{\circ} \mathrm{C}\right)$ is below $1 \mathrm{mV}$ for the longest stress time considered, and therefore the charge trapping component can be neglected in our study, focusing only on the hot carrier degradation. Table I summarizes the DUTs and test conditions: note in this study, we are considering the averaged-out impact of a sufficiently large interface state population, whereas the individual defect induced degradation under hot carrier stress is beyond the scope of this work, as it would require a description of the percolated channel conduction in ultra-scaled devices, as opposite to the continuous compact model used here.

Table. I : Summary of device technologies and stress conditions used in this work.

\begin{tabular}{|c|c|c|c|c|c|}
\hline & Gate-stack & EOT & w/ Leff $\left._{\text {ef }} / \mathrm{um}\right)$ & Stress $\left\{\mathrm{V}_{\mathrm{G}}, \mathrm{V}_{\mathrm{D}}\right\}$ & Stress $\mathrm{T}\left({ }^{\circ} \mathrm{C}\right)$ \\
\hline Device-A & HKMG & $1.3 \mathrm{~nm}$ & $1 / 0.075$ & $\begin{array}{c}\{1,1.8\},\{1.2,1.8\} \\
\{1.4,1.8\},\{1.6,1.8\}\end{array}$ & $25,75,125$ \\
\hline Device-B & SiON & $2.3 \mathrm{~nm}$ & $1 / 0.075$ & $\begin{array}{c}\{1.5,1.9\},\{1.7,1.9\} \\
\{1.9,1.9\},\{1.5,2.1\}\end{array}$ & $25,75,125$ \\
\hline
\end{tabular}

For the electrical characterization, the cycled stress-measure sequence shown in Fig. $\mathbf{5}$ is used: after the DC "stress" stage, the device's linear transfer characteristic is probed, followed by the charge pumping (CP) measurement using the base level sweep technique [19]. The base level of the CP trapezoidal waveform is swept from inversion to accumulation, whereas the amplitude is kept at $1.2 \mathrm{~V}$; the leading and trailing edges are kept at 50ns. The $\mathrm{CP}$ measurement within each "measure" stage will be referred to as "in-situ" $\mathrm{CP}$ (as opposed to the more thorough post-stress CP discussed next). Since the CP probed $N_{i t}$ is strongly dependent on the measurement temperature (i.e., the hot carrier stress temperature for the "in-situ" CP) and does not reflect the total $N_{i t}$ value, a post-stress temperature-resolved CP measurement (CP-T) is performed (referred below as "ex-situ") for extracting the temperature dependence of $N_{i t}$ which allows to estimate the total $N_{i t}$ and to rescale the "in-situ" CP results.

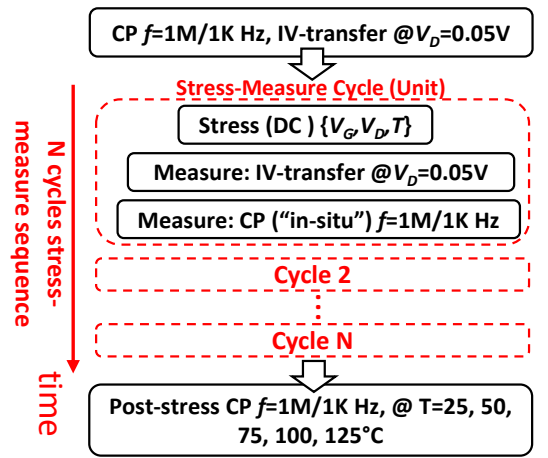

Fig. 5: A cycled stress-measure sequence is used for hot carrier degradation characterization. After the DC stress, both $\mathrm{I}-\mathrm{V}$ and $\mathrm{CP}$ measurements are performed in each "measure" stage. Up to $10^{4}$ seconds cumulative stress time is applied experimentally.

\section{Results ANd Discussion}

The wafer-level stress experiment is performed with the above-described measurement sequence (Fig. 5), covering the test devices and conditions listed in Table I. The measured I-V transfer characteristics are fitted with the EKV model. An example of the fitting results is plotted in Fig. 6-the linear transfer characteristic under strong inversion is accurately captured. The inset of Fig. $\mathbf{6}$ shows the time evolution of the fitted $K$ and $\Delta V_{t h}$ : a power-law $\left(y=k x^{n}\right)$ is used to describe the kinetics of both the extracted quantities. The discrepancy between the kinetics (i.e., time-exponents $n$ ) of the two quantities-despite them being manifestations of the same generated interface state profile-is due to the strong dependence of $\Delta V_{t h}$ on the underlying damage localization, as discussed in Section II-B. Furthermore, recalling Fig. 2, it is to be expected that the apparent $\Delta V_{t h}$ in the inset of Fig. 6 represents a (large) over-estimation of the actual $\Delta V_{t h}$ (intended as the pure electrostatic impact of a given interface charge density). It is important to point out that the $\Delta V_{t h}$ will also modify the mobility-gate voltage relation via the inversion carrier [ $n_{\text {inv }}$ in Eqs. (8-10)]; however, such secondary effect is small compared to the mobility degradation ratio $K$, especially for devices with gate-stack specifically optimized to minimize charge trapping under the relevant stress conditions, as done here. Based on the I-V fits, the modelled carrier mobility is plotted as a function of inversion carrier density $n_{\text {inv }}$ in Fig. 7, for increasing stress times.

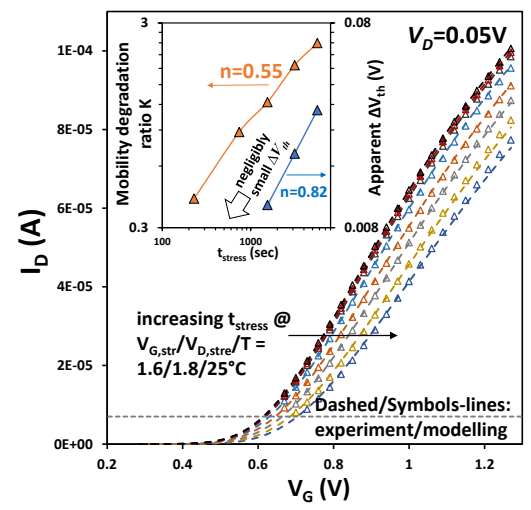

Fig. 6: Measured I-V curves (dashed lines) plotted against the calibrated compact model (symbols). For the aged I-V's, only the I-V curve under strong inversion is fitted and plotted. The inset shows the time evolution 
of the fitted quantities $\mathrm{K}$ (mobility degradation ratio) and $\Delta V_{t h}$. Note: as indicated by the bold arrow in the inset, the fitted short-term $\Delta V_{t h}$ is below the modelling resolution and is therefore neglected.

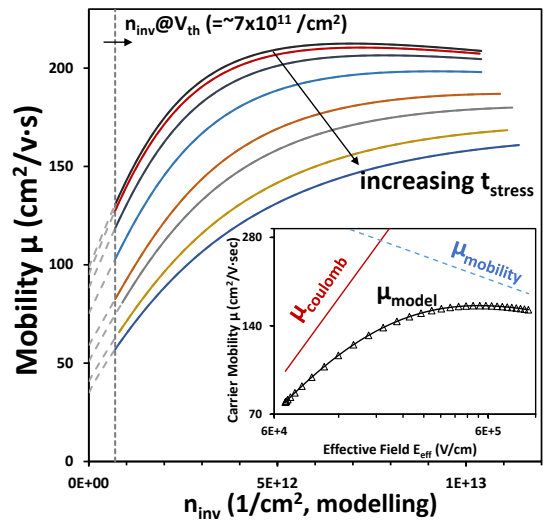

Fig. 7: The modelled mobility in device-A (corresponding to the I-V fits of Fig. 6) is plotted as a function of $n_{\text {inv }}$, for increasing stress times. The inset shows the limiting mechanism of carrier mobility in a pristine device under different effective field $E_{\text {eff }}$ using Eqs. (8-10): for low $E_{\text {eff, the }}$ mobility is limited by Coulomb scattering; with increasing $E_{\text {eff, }}$ the impact of Coulomb scattering is reduced.

Immediately after each I-V measurement, the "in-situ" CP measurement is performed; an example of the results is depicted in Fig. 8. The interface state density is evaluated from the peak substrate current $\left(I_{\text {sub }}=q A_{g} N_{i f}\right.$, where $A_{G}$ and $f$ are the effective gate area and CP frequency). However, as the "in-situ" CP measurement temperature is compliant to the stress temperature, the temperature dependence of the defect energy level that can be probed by $\mathrm{CP}$ measurement has to be considered. To account for this effect, a post-stress ("ex-situ") CP-T measurement is performed, where the temperature is swept from $25-125^{\circ} \mathrm{C}$ with a $25^{\circ} \mathrm{C}$-step. Note that a typical wafer chuck temperature change is finished within $\sim 10 \mathrm{~min}$, after which the CP-T measurement is performed again, the tight experimental timing control further suppresses any potential recovery of hot carrier induced interface state [24]. An example of the "ex-situ" CP-T measurement is shown in Fig. 9.

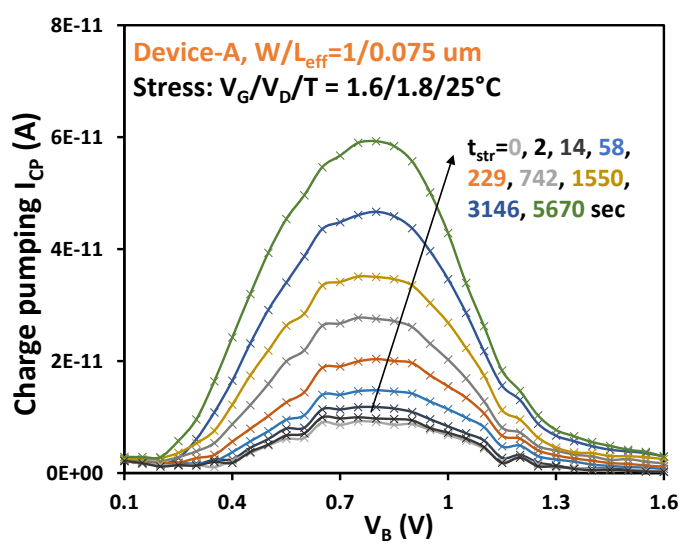

Fig. 8: "In-situ" base-level sweep CP measurement $[18,19]$ used to monitor the degradation kinetics. The CP current $I_{C P}$ is evaluated as the current difference between high-frequency $(f=1 \mathrm{MHz})$ and low frequency $(f=1 \mathrm{KHz})$ values, to exclude any contribution related to gate leakage.

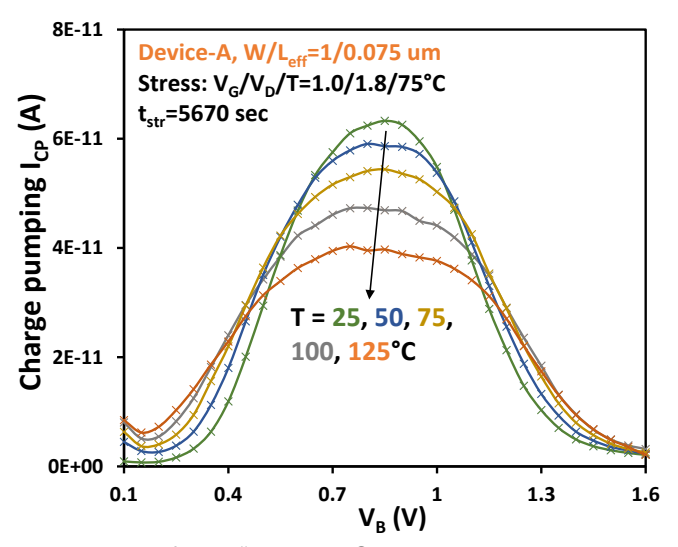

Fig. 9: An example of the "ex-situ" CP-T measurement result. As the measurement temperature increases, $I_{C P}$ decreases.

The "ex-situ" CP-T result is evaluated using the theory proposed in $[18,19]$, where the temperature dependence of the $\mathrm{CP}$ current has been described by Eq. (14), with the parameters $a, b, c$ defined in Eqs. (15-17):

$$
\begin{gathered}
I_{C P}(T)=-a T-b T \ln (T)+c \\
a=2 q k f A_{g} \overline{D_{l t}} \cdot \ln \left[\sqrt{\sigma_{n} \sigma_{p}} \sqrt{\frac{3 k}{m^{*}}} K_{i} \frac{\left|V_{t h}-V_{f b}\right|}{\Delta V_{g}} \sqrt{t_{r} t_{f}}\right] \\
b=2 q k f A_{g} \overline{D_{l t}} \\
c=q f A_{g} \overline{D_{l t}} E_{g}
\end{gathered}
$$

Note $\overline{D_{l t}}$ is the average interface state density $\left(1 / \mathrm{cm}^{2} \cdot \mathrm{eV}\right)$ and $E_{g}$ is the band gap of silicon. After fitting the experimental $I_{C P}$ with Eqs. (14-17), the interface state density $\left(1 / \mathrm{cm}^{2}\right)$ is calculated with Eq. (18). An example of this complete $N_{i t}$ extraction can be found in Fig. 10.

$$
N_{i t}=\overline{D_{l t}} \cdot E_{g}
$$

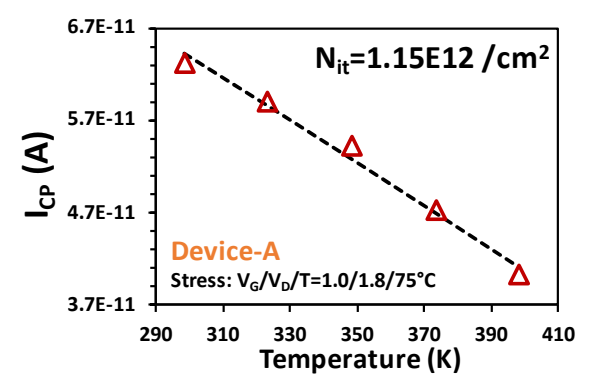

Fig. 10: Extracted $N_{i t}$ based on the "ex-situ" CP-T measurement, cf. Eqs. $(14-18)[18,19]$.

The ratio between $N_{i t}$ and $N_{i t, T}$ (where the latter represents the $N_{i t}$ estimated simply by the peak CP current at each temperature during the CP-T measurement) is calculated and summarized in Fig. 11. It is important to emphasize that the extraction of "Trescaling ratio" only involves the data collected during the "exsitu" CP-T measurement and does not involve any "in-situ" CP results. Such experimental design also helps in improving the robustness against potential recovery of hot carrier induced interface state [24], albeit it is observed to be negligible for the investigated devices. Since the CP-T measurement temperature is a super-set of the stress measurement temperature, only the extracted "T-rescaling ratio" is needed for rescaling the "insitu" CP measured interface state density. 

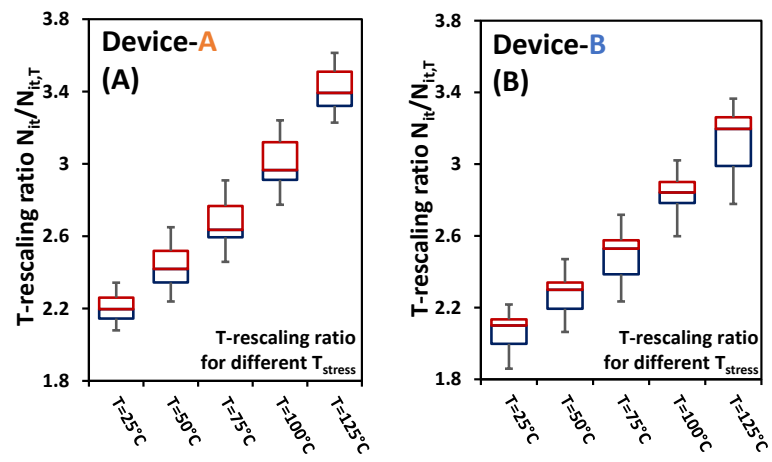

Fig. 11: Boxplot of all data points collected during the "ex-situ" CP-T measurement. Despite the similar trend in temperature dependence, the different "T-rescaling ratio" between device-A $(A)$ and device-B $(B)$ may come from the difference in electron and hole capture cross-sections $\left(\sigma_{\mathrm{n}}, \sigma_{\mathrm{p}}\right)$ in Eq. (15).

For each measured device, a single "T-rescaling ratio" can be extracted and used to correct the "in-situ" CP results. Based on these procedures, the rescaled $N_{i t}$ value ("with T-rescaling") is expected to reflect the total interface state density, i.e., not influenced by the hot carrier stress temperature, and should therefore closely correlate with the mobility degradation ratio $K$ as extracted from the I-V modelling. To examine this point, the time evolutions of the two quantities $\left(K, N_{i t}\right)$ are plotted in Fig. 12 and time-exponent $n$ is compared in Fig. 13 for all $\sim 32$ devices that were measured: the two exponents, as extracted using two independent approaches, shows a relative difference no larger than $10 \%$. Such extensive match clearly justifies the experimental design discussed above and enables further numerical treatments.

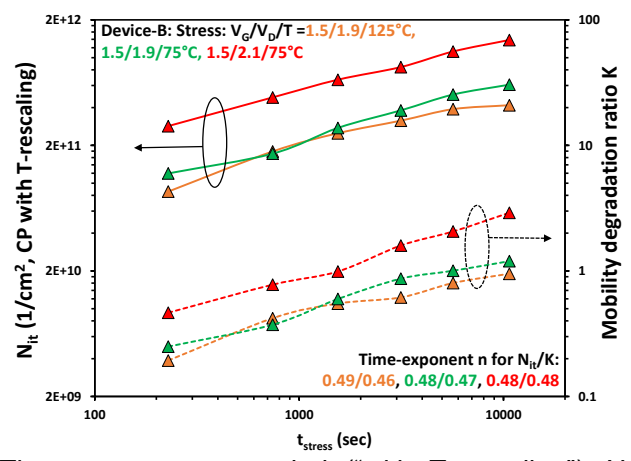

Fig. 12: The temperature-rescaled ("with T-rescaling") $N_{i t}$ from $\mathrm{CP}$ measurement and the mobility degradation ratio $K$ from $\mathrm{I}-\mathrm{V}$ modelling are plotted versus the stress time for 3 stress conditions as examples. The power-law time-exponent $n$ is fitted and summarized in Fig. 13. The $N_{i t, \text { ref }}\left(=N_{i t} / K\right)$ as defined in Eqs. $(11-12)$ can be further extracted based on these data.

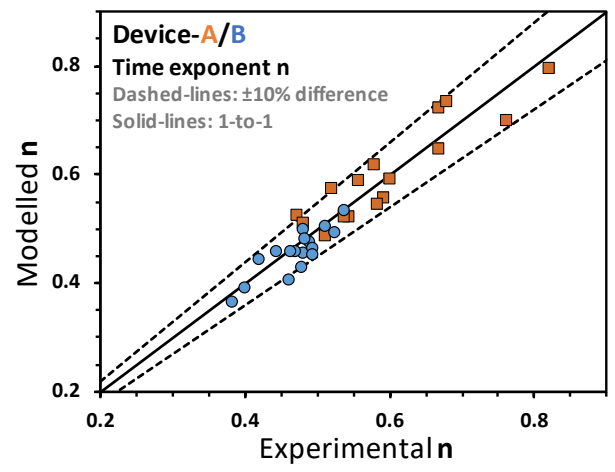

Fig. 13: Comparison between the experimental $n$, i.e., time-exponent from the CP measured $N_{i t}$, and the modelled $n$, i.e., time exponent from the modelled I-V mobility degradation ratio $K$.

By taking the ratio between $N_{i t}$ and $K$, as defined in Eqs. (1112), the mobility degradation normalization parameter $N_{i, t, r e}$ can be extracted for each measured device and each stress time. In order to feed back the CP results to the I-V model, as well as to demonstrate the consistency between the CP-measured $N_{i t}$ ("with T-rescaling") and the I-V modelled $N_{i t}$ (via $K$ ratio), it is important to examine the various dependences of $N_{i t, \text { ref }}$, i.e., the EOT, the temperature, and the degradation dependence. For this purpose, the $N_{i t, r e f}$ vs. stress measurement temperature for both device types are plotted in Fig. 14-no systematic dependence upon EOT or temperature can be observed. Furthermore, as visualized in Fig. 15, no consistent degradation dependence is observed, i.e., the normalization parameter $N_{i, \text { ref }}$ does not change with increasing interface state density. Therefore, regarding the mobility degradation normalization parameter $N_{i t, \text { ref }}$, a constant median value of $\sim 4.1 \times 10^{11} / \mathrm{cm}^{2}$, with a standard deviation of $\sim 4.7 \times 10^{10} / \mathrm{cm}^{2}$, is reported based on the broad experimental evidence discussed in this work. Based on the established $N_{i, \text { ref }}$ value, it is possible to accurately evaluate the integrated stress-induced $N_{i t}$ based on the compact model of the device I-V, or conversely to convert a given stress-induced $N_{i t}$ into mobility (and therefore current) degradation.

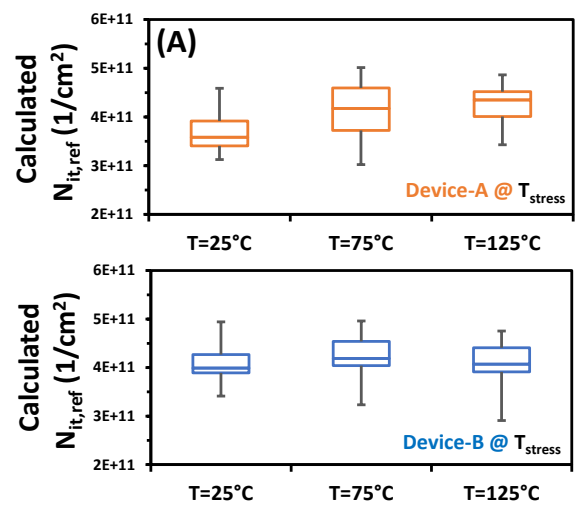

Fig. 14: The EOT dependence [by separating the two device types: (A) device-A; $(B)$ device-B] and the temperature dependence are checked by categorizing the extracted $N_{i, \text { ref }}$. no systematic EOT or temperature dependence is observed. Note: the temperatures listed above refer to the stress temperatures (and not the temperature of the "ex-situ" CP-T measurement).

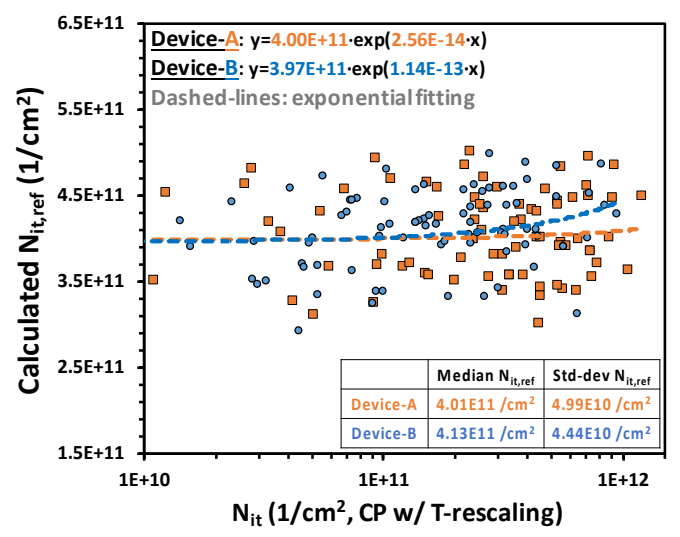

Fig. 15: The degradation dependence of $N_{i t, r e f}$ is examined: despite the small upwards tail observed in device-B for very large stress-induced 
interface state density (which deviates by just $\sim 10 \%$ from its median value), no systematic degradation dependence of $N_{i t, \text { ref }}$ is observed. A constant median value of $N_{i, \text { ref }}=\sim 4.1 \times 10^{11} / \mathrm{cm}^{2}$ with a small standard deviation of $\sim 4.7 \times 10^{10} / \mathrm{cm}^{2}$ is therefore reported.

\section{SUMMARY}

In this work, a comprehensive investigation of the impact of the hot carrier induced interface state on carrier mobility in nMOSFET has been performed. The impact of localization effect under hot carrier stress has been investigated, which indicated that the mobility degradation ratio is not influenced by the localization effect and therefore can be directly probed experimentally and correlated to the total integrated $N_{i t}$. The EKV-based I-V model and the charge pumping measurement were used as independent techniques for probing the interface state density in aged devices. A good agreement in the timeevolution of the degradation as assessed by the two independent techniques was demonstrated, validating the effectiveness of the modelling equations in translating a physical interface defect density into the corresponding carrier mobility. Furthermore, by sampling across a broad range of stress voltages, stress temperatures, and different device technologies, a single mobility degradation normalization parameter $\left(N_{i t, r e f}\right.$ $=\sim 4.1 \times 10^{11} / \mathrm{cm}^{2}$ ) was reported, with negligible EOTs, stress temperatures or device degradation level-dependences. Based on the combined experimental techniques, and supported by the consistency across a broad range of experimental conditions, the extracted mobility degradation normalization parameter $N_{i t, r e f}$ is expected to act as an universal constant in the commonly used I-V compact models, to bring improved consistency between the interface state density as estimated from the I-V compact model and the physical interface state density actually present in an aged device.

\section{REFERENCES}

[1] Novak, S., C. Parker, D. Becher, M. Liu, M. Agostinelli, M. Chahal, P. Packan, P. Nayak, S. Ramey, and S. Natarajan, "Transistor aging and reliability in 14nm tri-gate technology," 2015 IEEE International Reliability Physics Symposium, Monterey, CA, 2015, pp. 2F.2.1-2F.2.5, doi: 10.1109/IRPS.2015.7112692.

[2] A. Rahman, J. Dacuna, P. Nayak, G. Leatherman and S. Ramey, "Reliability studies of a 10nm high-performance and low-power CMOS technology featuring 3rd generation FinFET and 5th generation HK/MG," 2018 IEEE International Reliability Physics Symposium (IRPS), Burlingame, CA, 2018, pp. 6F.4-1-6F.4-6, doi: 10.1109/IRPS.2018.8353648.

[3] M. Bina et al., "Predictive Hot-Carrier Modeling of n-Channel MOSFETs," in IEEE Transactions on Electron Devices, vol. 61, no. 9, pp. 3103-3110, Sept. 2014, doi: 10.1109/TED.2014.2340575.

[4] S. Reggiani, G. Barone, S. Poli, E. Gnani, A. Gnudi, G. Baccarani, M.Y. Chuang, W.D. Tian, R. Wise, "TCAD Simulation of Hot-Carrier and Thermal Degradation in STI-LDMOS Transistors," in IEEE Transactions on Electron Devices, vol. 60, no. 2, pp. 691-698, Feb. 2013, doi: 10.1109/TED.2012.2227321.

[5] Z. Wu, J. Franco, P.J. Roussel, S. Tyaginov, B. Trujen, M. Vandemale, G. Hellings, N. Collaert, G. Groeseneken, D. Linten, B. Kaczer, "A physics-aware compact modeling framework for transistor aging in the entire bias space," 2019 IEEE International Electron Devices Meeting (IEDM), San Francisco, CA, USA, 2019, pp. 21.2.1-21.2.4, doi: 10.1109/IEDM19573.2019.8993603.

[6] S. Tyaginov et al., "A Compact Physics Analytical Model for Hot-Carrier Degradation," 2020 IEEE International Reliability Physics Symposium (IRPS), Dallas, TX, USA, 2020, pp. 1-7, doi: 10.1109/IRPS45951.2020.9128327.

[7] I. Meric, S. Ramey, S. Novak, S. Gupta, S. P. Mudanai and J. Hicks, "Modeling Framework for Transistor Aging Playback in Advanced Technology Nodes," 2020 IEEE International Reliability Physics Symposium (IRPS), Dallas, TX, USA, 2020, pp. 1-6, doi: 10.1109/IRPS45951.2020.9129601.
[8] S. C. Sun and J. D. Plummer, "Electron Mobility in Inversion and Accumulation Layers on Thermally Oxidized Silicon Surfaces," in IEEE Journal of Solid-State Circuits, vol. 15, no. 4, pp. 562-573, Aug. 1980, doi: 10.1109/JSSC.1980.1051439.

[9] F. Hsu and S. Tam, "Relationship between MOSFET degradation and hotelectron-induced interface-state generation," in IEEE Electron Device Letters, vol. 5, no. 2, pp. 50-52, Feb. 1984, doi: 10.1109/EDL.1984.25829.

[10] H.S. Wong, M.H. White, T.J. Krutsick, R.V. Booth, "Modeling of transconductance degradation and extraction of threshold voltage in thin oxide MOSFET's." Solid-State Electronics 30.9 (1987): 953-968. https://doi.org/10.1016/0038-1101(87)90132-8

[11] Y. Leblebici and S. -. Kang, "Modeling of nMOS transistors for simulation of hot-carrier-induced device and circuit degradation," in IEEE Transactions on Computer-Aided Design of Integrated Circuits and Systems, vol. 11, no. 2, pp. 235-246, Feb. 1992, doi: 10.1109/43.124402.

[12] P. Sharma et al., "Modeling of Hot-Carrier Degradation in nLDMOS Devices: Different Approaches to the Solution of the Boltzmann Transport Equation," in IEEE Transactions on Electron Devices, vol. 62, no. 6, pp. 18111818, June 2015, doi: 10.1109/TED.2015.2421282.

[13] M. Jech, G. Rott, H. Reisinger, S. Tyaginov, G. Rzepa, A. Grill, D. Jabs, C. Jungemann, M. Waltl, and T. Grasser, "Mixed Hot-Carrier/Bias Temperature Instability Degradation Regimes in Full \{VG, VD\} Bias Space: Implications and Peculiarities," in IEEE Transactions on Electron Devices, vol. 67, no. 8, pp. 3315-3322, Aug. 2020, doi: 10.1109/TED.2020.3000749.

[14] B. Ullmann, M. Jech, K. Puschkarsky, G. A. Rott, M. Waltl, Y. Illarionov, H. Reisinger, and T. Grasser, "Impact of Mixed Negative Bias Temperature Instability and Hot Carrier Stress on MOSFET Characteristics-Part II: Theory," in IEEE Transactions on Electron Devices, vol. 66, no. 1, pp. 241248, Jan. 2019, doi: 10.1109/TED.2018.2873421.

[15] B. Ullmann, M. Jech, K. Puschkarsky, G. A. Rott, M. Waltl, Y. Illarionov, H. Reisinger, and T. Grasser, "Impact of Mixed Negative Bias Temperature Instability and Hot Carrier Stress on MOSFET Characteristics-Part I: Experimental," in IEEE Transactions on Electron Devices, vol. 66, no. 1, pp. 232-240, Jan. 2019, doi: 10.1109/TED.2018.2873419.

[16] C.C. Enz, E. A. Vittoz, Charge-based MOS transistor modeling: the EKV model for low-power and RF IC design. John Wiley \& Sons, 2006.

[17] C.C. Enz, F. Krummenacher and E. A. Vittoz, "An analytical MOS transistor model valid in all regions of operation and dedicated to low-voltage and low-current applications." Analog integrated circuits and signal processing 8.1 (1995): 83-114. https://doi.org/10.1007/BF01239381

[18] G. Van den bosch, G. V. Groeseneken, P. Heremans and H. E. Maes, "Spectroscopic charge pumping: A new procedure for measuring interface trap distributions on MOS transistors," in IEEE Transactions on Electron Devices, vol. 38, no. 8, pp. 1820-1831, Aug. 1991, doi: 10.1109/16.119021.

[19] G. Groeseneken, H. E. Maes, N. Beltran and R. F. De Keersmaecker, "A reliable approach to charge-pumping measurements in MOS transistors," in IEEE Transactions on Electron Devices, vol. 31, no. 1, pp. 42-53, Jan. 1984, doi: 10.1109/T-ED.1984.21472.

[20] B. Ruch, G. Pobegen and T. Grasser, "Investigation of the Temperature Dependence of Hot-Carrier Degradation in Si MOSFETs Using Spectroscopic Charge Pumping," in IEEE Transactions on Electron Devices, vol. 67, no. 10, pp. 4092-4098, Oct. 2020, doi: 10.1109/TED.2020.3018091.

[21] S. E. Rauch and G. La Rosa, "The energy-driven paradigm of NMOSFET hot-carrier effects," in IEEE Transactions on Device and Materials Reliability, vol. 5, no. 4, pp. 701-705, Dec. 2005, doi: 10.1109/TDMR.2005.860560.

[22] K. Chain, J.H. Huang, J. Duster, P. K. Ko and C. M. Hu, "A MOSFET electron mobility model of wide temperature range $(77-400 \mathrm{~K})$ for IC simulation." Semiconductor science and technology 12.4 (1997): 355. PII: S0268-1242(97)78841-2

[23] Restrepo, O. D., K. Varga, and S. T. Pantelides. "First-principles calculations of electron mobilities in silicon: Phonon and Coulomb scattering." Applied Physics Letters $94.21 \quad$ (2009): 212103. https://doi.org/10.1063/1.3147189

[24] M. Vandemaele, J. Franco, S. Tyaginov, G. Groeseneken and B. Kaczer, "Modeling of Repeated FET Hot-Carrier Stress and Anneal Cycles Using Si-H Bond Dissociation/Passivation Energy Distributions," in IEEE Transactions on Electron Devices, vol. 68, no. 4, pp. 1454-1460, April 2021, doi: 10.1109/TED.2021.3061025. 